\title{
REFERENCES
}

1. R. Godement, Analyse harmonique dans les groupes centraux II, C. R. Acad. Sci. Paris vol. 225 (1947) pp. 221-223.

2. F. I. Mautner, Structure of the regular representation of certain discrete groups, To appear in Duke Math. J.

3. - The completeness of the irreducible unitary representations of a locally compact group, Proc. Nat. Acad. Sci. U.S.A. vol. 34 (1948) pp. 52-54.

4. - Unitary representations of locally compact groups I, Ann. of Math. vol. 51 (1950) pp. 1-25.

5. J. von Neumann, On rings of operators. Reduction theory, Ann. of Math. vol. 50 (1949) pp. 401-485.

6. J. von Neumann and F. J. Murray, On rings of operators IV, Ann. of Math. vol. 44 (1943) pp. 716-808.

MassachusetTs Institute of Technology

\section{A NOTE ON THE CHARACTERISTIC POLYNOMIALS OF CERTAIN MATRICES}

\section{WILLIAM T. REID}

The purpose of this note is to establish the following theorem on matrices with elements in an arbitrary field $\mathfrak{F}^{1}{ }^{1}$ In particular, $I$ denotes the $n \times n$ identity matrix; 0 is used for the zero matrix, square or rectangular, its dimensions being clear in each case from the context. The determinant of an $n \times n$ matrix $M(\lambda)$ with elements in $\mathfrak{F}(\lambda)$, the ring of polynomials in $\lambda$ with coefficients in $\mathfrak{F}$, is denoted by $|M(\lambda)|$; in particular, if $M$ is an $n \times n$ matrix with elements in $\mathfrak{F}$, then $|\lambda I-M|$ is the characteristic polynomial of $M$.

TheOREM. If $A$ is an $n \times m$ matrix, and $D$ is an $n \times n$ matrix, then in order that $|\lambda I-A B|=|\lambda I-A B-D|$ for arbitrary $m \times n$ matrices $B$ it is necessary and sufficient that $D$ be nilpotent and $D A=0$. In particular, if $C$ is an $m \times n$ matrix, then in order that $|\lambda I-A B|$ $=|\lambda I-A(B+C)|$ for arbitrary $m \times n$ matrices $B$ it is necessary and sufficient that $A C A=0$.

Presented to the Society, September 2, 1949; received by the editors April 5, 1949 and, in revised form, July 23, 1949.

${ }^{1}$ In the original manuscript of this paper attention was restricted to $\mathfrak{F}$ the field of complex numbers. The proof there given of the fact that the nilpotency of $D$ and $D A=0$ imply $|\lambda I-A B|=|\lambda I-A B-D|$, for arbitrary $B$, was valid for arbitrary fields $\mathfrak{F}$. The proof here given of the necessity of $D A=0$ for $\mathfrak{F}$ an arbitrary field was suggested by the referee. 
An alternate proof of the sufficiency part of the statement in the last sentence of the theorem has been obtained recently by Parker, ${ }^{2}$ in generalizing an earlier result of A. Brauer. ${ }^{3}$

The principal tool used in the proof of the above theorem is the fact that a matrix $D$ is nilpotent if and only if $\lambda^{n}=|\lambda I-D|$. That $\lambda^{n}=|\lambda I-D|$ implies the nilpotency of $D$ follows from the CayleyHamilton theorem. On the other hand, if $D$ is nilpotent of index $s$, then $\left|\lambda I-D^{r}\right|=\lambda^{n}$ for $r \geqq s$, while if $\lambda^{n}=\left|\lambda I-D^{r}\right|$ for $r \geqq t+1>1$, then $\left|\lambda I-D^{t}\right| \cdot\left|\lambda I+D^{t}\right|=\left|\lambda^{2} I-D^{2 t}\right|=\left|\lambda^{2} I\right|=\lambda^{2 n}$, so that $\left|\lambda I-D^{t}\right|$ $=\lambda^{n}$, and by induction $|\lambda I-D|=\lambda^{n}$.

The sufficiency part of the statement in the first sentence of the above theorem is established by noting that if $D$ is nilpotent and $D A=0$, then $\lambda^{n}|\lambda I-A B|=|(\lambda I-D)(\lambda I-A B)|=\left|\lambda^{2} I-\lambda(A B+D)\right|$ $=\lambda^{n}|\lambda I-A B-D|$, and $|\lambda I-A B|=|\lambda I-A B-D|$ for arbitrary $m \times n$ matrices $B$.

On the other hand, suppose that $|\lambda I-A B|=|\lambda I-A B-D|$ for all $m \times n$ matrices $B$. The choice $B=0$ gives $|\lambda I-D|=\lambda^{n}$, and hence the nilpotency of $D$. Then $\left|\lambda^{2} I-\lambda(A B+D)\right|=\lambda^{n}|\lambda I-(A B+D)|$ $=|\lambda I-D| \cdot|\lambda I-A B|=\left|\lambda^{2} I-\lambda(A B+D)+D A B\right|$ for arbitrary $m$ $\times n$ matrices $B$. Now if $D A=K=\left\|K_{i \beta}\right\|(i=1, \cdots, n ; \beta=1, \cdots, m)$ has a nonzero element $K_{p q}$, then the choice $B_{0}=\left\|\delta_{\alpha q} \delta_{p j} K_{p q}^{-1}\right\|(\alpha$ $=1, \cdots, m ; j=1, \cdots, n)$ gives $D A B_{0}=G=\left\|G_{i j}\right\|(i, j=1, \cdots, n)$ with $G_{i j}=0$ if $j \neq p, G_{p p}=1$. If $A B_{0}+D=H=\left\|H_{i j}\right\|(i, j=1, \cdots, n)$, then from the above, $\left|\lambda^{2} I-\lambda H\right|=\left|\lambda^{2}-\lambda H+G\right|$. This is impossible, however, since $\left|\lambda^{2} I-\lambda H+G\right|=\left|\lambda^{2} I-\lambda H\right|+|R(\lambda)|$, where $R_{i j}(\lambda)$ $=\lambda^{2} \delta_{i j}-\lambda H_{i j}$ if $j \neq p, R_{i p}(\lambda)=G_{i p}$, and consequently $|R(\lambda)|$ is a nonzero polynomial $\lambda^{2 n-2}+$ terms of lower degree. Hence $D A=0$, and the statement of the first sentence of the theorem is.proved.

The second sentence of the theorem follows from the first part, together with the remark that if $D=A C$, then $0=D A=A C A$ implies $0=D A C=D^{2}$ so that $D$ is nilpotent.

\section{NORTHWESTERN UNIVERSITY}

${ }^{2} \mathrm{~W}$. V. Parker, On the characteristic equations of certain matrices, Bull. Amer. Math. Soc. vol. 55 (1949) pp. 115-116.

${ }^{3}$ Alfred Brauer, On the characteristic equations of certain matrices, Bull. Amer. Math. Soc. vol. 53 (1947) pp. 605-607. 